\title{
Evaluating Common Variants in NOS1AP in Patients with Implantable Cardioverter Defibrillator for Secondary Prevention
}

\section{Xiaobiao Zang}

Fuwai Central China Cardiovascular Hospital https://orcid.org/0000-0003-0872-5172

\section{Zhang Shulong}

Affiliated Zhongshan Hospital of Dalian University

\section{Sisi Li}

Gannan Medical University

\section{Xianqing Wang}

Fuwai Central China Cardiovascular Hospital

\section{Weifeng Song}

Fuwai Central China Cardiovascular Hospital

\section{Ke Chen}

Fuwai Central China Cardiovascular Hospital

\section{Jifang Ma}

Fuwai Central China Cardiovascular Hospital

\section{Xin Tu}

Huazhong University of Science and Technology

Yunlong Xia

First Affiliated Hospital of Dalian Medical University

\section{Zhao Yonghui}

Fuwai Central China Cardiovascular Hospial

Gao Chuanyu ( $\sim$ gaochuanyu01@163.com )

People's Hospital of Zhengzhou University https://orcid.org/0000-0002-5817-5212

\section{Research article}

Keywords: Nitric oxide synthase 1 adaptor protein gene, Implantable cardioverter defibrillator, QT interval, Sudden cardiac death, Single nucleotide polymorphism

Posted Date: September 22nd, 2020

DOI: https://doi.org/10.21203/rs.3.rs-70808/v1

License: (c) (i) This work is licensed under a Creative Commons Attribution 4.0 International License. Read Full License 


\section{Abstract \\ Background}

Contemporary researchers found single nucleotide polymorphisms (SNPs) in nitric oxide synthase 1 adaptor protein (NOS1AP) gene are associated with altered QT intervals and SCD. However, the clinical utility and implications of these SNP have not been described. This study aims to explore the clinical utility and implications of SNPs in nitric oxide synthase 1 adaptor protein (NOS1AP) in patients with implantable cardioverter defibrillator (ICD) for secondary prevention.

\section{Methods}

We firstly conducted a case-control study to evaluate the associations between hot-spot SNPs in NOS1AP (rs12143842, rs10494366, rs12567209 and rs16847548) and patients with ICD for secondary prevention. Then the clinical values of the positive SNPs were further evaluated in these patients. All patients were divided into three groups according to different genotypes of the positive SNPs. ICD interrogation data at 3, 12 months, and 3 years after implantation, which include rapid ventricular arrhythmia episodes and appropriate therapies, were analyzed in three genotypes.

\section{Results}

Case-control study revealed significant allelic association between rs10494366 and ICD recipients who experienced appropriate therapies. After a mean follow-up time of $31.70 \pm 9.15$ months, we detected not only significant difference among three genotypes on the distribution of ICD shocks and appropriate therapies, but apparently also the correlation of rs 10494366 and ICD shocks. Furthermore, under kaplan-meier and cox regression analysis, TT genotype both showed higher risk for sudden cardiac death (SCD) compared with GG genotype.

\section{Conclusions}

The present study revealed that SNP rs10494366 was associated with appropriate therapies and SCD in patients with ICD for secondary prevention for the first time.

\section{Background}

Sudden cardiac death (SCD) is a common clinical form of death and presumably due to a cardiac arrhythmia or hemodynamic catastrophe. Robust data supported implantable cardioverter defibrillation (ICD) is highly effective in terminating life-threatening ventricular arrhythmia (VA). ICD has been applied in various patients including survivors of cardiac arrest, patients with VT and structural heart disease, and patients with significant LV dysfunction, for more than 3 decades. Recently, many studies found allelic variants in nitric oxide synthase 1 adaptor protein (NOS1AP) gene, which encodes a cytosolic ligand of neuronal nitric oxide synthase, are associated with altered QT intervals and SCD [1-4]. Among these allelic variants, rs12143842, rs10494366, rs12567209 and rs16847548 have attracted the most attention. However, the clinical utility and implications of these hot-spot SNPs have not been described. We firstly conducted a case-control study in patients receiving ICD for secondary prevention and healthy people aim to find the relation between these SNPs and patients with ICD. And then an evaluation of the relativity of positive SNPs and ICD events was performed to further explore its clinical value in ICD recipients.

\section{Materials And Methods}

\section{Subjects}

From September 2012 to June 2015, 97 consecutive patients underwent implantation of ICD for secondary prevention in Zhengzhou University People's Hospital. 348 healthy people were recruited from physical examination center of our hospital as 
control group. The treatment of enrolled patients conformed to expert consensus on secondary prevention indications [5]. Secondary prevention was defined if the patient has survived a cardiac arrest or experienced rapid ventricular arrhythmia (RVA) including sustained ventricular tachycardia (VT) and ventricular fibrillation (VF). Exclusion criteria were as follows: 1) expectation of life was less than 1 year; 2) patients suffered pacemaker infections that need to be removed; 3) pacemaker malfunction or lead dislodgement. 9 patients were excluded after evaluated by clinicians ( 5 patients with a life expectancy less than 1 year, 2 patients suffered from pacemaker infections, and 2 patients had electrode displacement). 86 patients were enrolled in the present study finally. The research protocol was approved by the institutional review board of the Zhengzhou University People's Hospital. All participants provided informed written consent in accordance with the Declaration of Helsinki prior to enrollment.

A detailed clinical characteristic, including body mass index, drug therapy, electrocardiology and echocardiography parameters were collected at baseline. Coronary heart disease (CHD) was defined by coronary angiograph showing more than $75 \%$ stenosis in at least one main vessel of the coronary artery. Ischemic cardiomyopathy was defined as left ventricular dysfunction with Left ventricular enlargement caused by previous myocardial infarction or significant CHD [6]. The diagnosis of Dilated cardiomyopathy (DCM), Hypertrophic cardiomyopathy (HCM), and arrhythmogenic right ventricular cardiomyopathy (ARVC) were based on the classification scheme presented by the European Society of Cardiology [7]. SCD was defined as a witnessed natural death attributable to cardiac causes within 1 hour of onset of acute symptoms or as an unwitnessed unexpected death of a person seen in a stable medical condition within 24 hours before death without evidence of a noncardiac cause [8]. SCD event was adjudicated independently by two investigators. A third investigator independently reviewed the event to provide final classification if disagreement existed. All healthy people were ruled out the cardiovascular disease mentioned above.

\section{Genotyping}

Blood samples were collected from participants using tubes containing EDTA and genomic DNA was isolated from whole blood using the Wizard Genomic DNA Purification Kit (Promega, Shanghai, China). Genotyping of four common polymorphisms (rs12143842, rs10494366, rs12567209 and rs16847548) was performed on all cases and controls using polymerase chain reaction (PCR) and direct DNA sequencing. The primer sequences for PCR were designed by Primer3web version 4.0.0 (http://bioinfo.ut.ee/primer3/) and listed in Additional file 1. PCR was performed in a total reaction volume of $25 \mu \mathrm{L}$ containing $12.5 \mu \mathrm{L}$ of $2 \times$ Es Taq MasterMix (cwbio, Shanghai, CHINA), $0.5 \mu \mathrm{L}$ of each primer, $1.5 \mu \mathrm{L}$ of DNA template, and $10 \mu \mathrm{L}$ of $\mathrm{ddH}_{2} 0$ Water. The multiplex-touchdown PCR amplification protocol consisted of $5 \mathrm{~min}$ at $94^{\circ} \mathrm{C}$ for initial denaturation, 10 cycles of denaturing at $95^{\circ} \mathrm{C}$ for $30 \mathrm{~s}$, annealing at $65^{\circ} \mathrm{C}$ (with $1^{\circ} \mathrm{C}$ decrements from $65^{\circ} \mathrm{C}$ to $55^{\circ} \mathrm{C}$ at every cycle) for $30 \mathrm{~s}$, and extension at $72^{\circ} \mathrm{C}$ for $30 \mathrm{~s}$. This was followed by a further 30 cycles of denaturing at $94^{\circ} \mathrm{C}$ for $30 \mathrm{~s}$, annealing at $55^{\circ} \mathrm{C}$ for $30 \mathrm{~s}$, and extension at $72^{\circ} \mathrm{C}$ for $30 \mathrm{~s}$. The reaction was finished with a final extension for $5 \mathrm{~min}$ at $72^{\circ} \mathrm{C}$. PCR products for SNPs were direct DNA Sanger sequenced using the ABI 3730XL (Applied Biosystems, Foster City, CA, USA) after PCR amplification. Chromas 2.6.4 software (Technelysium Pty Ltd, South Brisbane, QLD, Australia) was used to view the DNA sequences.

\section{ICD programming and follow-up}

ICD programming was conducted in Henan Provincial Pacemaker Programing Center in our hospital. Recognition program of tachycardia was divided into 3 zones as following: VF zone with tachycardia circumference length (CL) less than 250ms, fast VT zone from $250 \mathrm{~ms}$ to $320 \mathrm{~ms}$, and slow VT zone from $321 \mathrm{~ms}$ to $400 \mathrm{~ms}$. The diagnose of VT zone based on the stability and morphological standard of tachycardia. The algorithms for discrimination of supraventricular tachycardia were activated in the VT zones. When tachycardia CL coincided slow VT zone, anti-tachycardia pacing (ATP) conducted at the $85 \%$ CL of VT for three tracings in the form of 8 consecutive pulses. And for fast VT zone, treatment procedure was ATP at the $85 \%$ CL of VT for three tracings in the form of 8 consecutive pulses, and shocks following if ATP failure. For VF zone, only one tracing ATP followed by shocks. Follow-up started at the time of ICD implantation and device interrogations were scheduled in pacemaker programing center every 3-6 months after ICD implantation. The longest follow-up time was 36 months. Pacemaker programming was required when patients experienced symptoms like syncope, incessant palpitations, or ICD therapy. All device interrogations were independently reviewed by the third-party technician to determine whether ICD therapies were delivered appropriately or inappropriately. Appropriate therapies included those delivered in response to VT, VF. Inappropriate therapies were defined as ATP or shock for supraventricular tachycardia, non-sustained VT, and oversensing events resulted from ICD lead noise, myopotentials, electromechanical interference, and T-wave. Intracardiac electrograms were reanalyzed by at least 2 independent

Page 3/14 
electrophysiologists to confirm the type of arrhythmia if episodes controversy exists. The parameters of the ICD were changed whenever necessary as dictated by the physicians during each follow-up. Participants were censored at the time of loss to followup or death if the cause of death was other than SCD.

\section{Statistical analyses}

Statistical analyses were performed using SPASS17.0 (SPSS Inc, Chicago, IL). The allelic association and genotypic association under dominant and recessive models were analyzed using a $2 \times 2$ chi-square tests. Data of baseline clinical characteristic was grouped by genotypes of the positive SNP. Continuous variables were expressed as mean \pm standard deviation. One-way ANOVA was performed for comparison between three continuous variables. Categorical data was counted and expressed as percentages and $c^{2}$ test was used to compare the difference among them. Frequencies of rapid ventricular arrhythmia episodes, ATPS and ICD shocks 3 months, 12 months after implantation, and at the end of follow-up time in three genotypes were figured out and compared using one-way ANOVA and bivariate correlation separately. Bivariate correlation of these variables and genotypes were evaluated by spearman correlation coefficient. The ratio of patients suffered from VA episodes and ICD therapies under different genotype model were compared by chi-square test. Fisher's exact test should be used instead of the chi-square test when any expected frequency is less than 1 or $20 \%$ of expected frequencies are less than or equal to 5 . Kaplan-meier curve of the cumulative probability of survival classified by genotypes of the positive variant was further performed using Log Rank method to analyze the impact of three genotypes on SCD. Finally, hazard ratios of different genotypes for SCD were calculated by cox regression analysis in complicate model using Forward: LR method. A $P$-value of $<0.05$ was considered statistically significant.

\section{Results}

\section{Patient characteristics and allelic associations}

A total of 86 patients and 348 control subjects were enrolled in this study. There were 39 patients with CHD, 34 patients with DCM and 8 cases of HCM, 3 cases of ARVC, 2 cases of long QT syndrome. Over a median follow-up of $31.70 \pm 9.15$ months, 32 ICD recipients experienced appropriate therapies and 24 patients dead. The baseline characteristic of patients were shown in Table 1. Genotype distribution of the four SNPs in controls were all in accordance with HWE. Significant allelic association was detected between rs10494366 and ICD recipients who suffered appropriate therapies $(P=0.024, \mathrm{OR}=1.25)$, while the association with ICD patients were not significant (Table 2). Association analysis of rs12143842, rs12567209 and rs16847548 under allele, dominant, recessive model did not show significant. 
Table 1

Baseline characteristics of patients with ICD for secondary prevention.

\begin{tabular}{|l|l|}
\hline Age, $y$, mean \pm SD & $53.55 \pm 12.28$ \\
\hline Female, $n(\%)$ & $41(47.67)$ \\
\hline Body mass index, $\mathrm{kg} / \mathrm{m}^{2}$, mean $\pm \mathrm{SD}$ & $28.46 \pm 5.08$ \\
\hline Coronary heart disease, $\mathrm{n}(\%)$ & $39(45.35)$ \\
\hline Dilated cardiomyopathy, $\mathrm{n}(\%)$ & $34(39.53)$ \\
\hline Other cardiovascular disease, $\mathrm{n}(\%)$ & $13(15.12)$ \\
\hline Hypertension, $\mathrm{n}(\%)$ & $37(43.02)$ \\
\hline Diabetes mellitus, $\mathrm{n}(\%)$ & $29(33.72)$ \\
\hline Treatment, $\mathrm{n}(\%)$ & \\
\hline ACEI/ARB & $44(51.16)$ \\
\hline Beta-blocker & $41(47.67)$ \\
\hline Amiodarone & $35(40.70)$ \\
\hline Electrocardiology parameters & \\
\hline CLBBB, $\mathrm{n}(\%)$ & $43(50.00)$ \\
\hline QRS duration, ms, mean \pm SD & $133.94 \pm 24.70$ \\
\hline LVEF(\%), mean \pm SD & $38.99 \pm 10.60$ \\
\hline $\begin{array}{l}\text { ACEl: Angiotensin-Converting Enzyme Inhibitors; ARB: Angiotensin Receptor Blockers; CLBBB: Complete Left Bundle Branch } \\
\text { Block; LVEF: left ventricular ejection fraction }\end{array}$ & \\
\hline
\end{tabular}


Table 2

Association analysis of four SNPs in NOS1AP between patients with ICD, patients suffered from appropriate therapies and controls.

\begin{tabular}{|c|c|c|c|c|c|c|c|c|c|c|c|c|c|}
\hline \multirow[t]{2}{*}{ SNP } & \multirow[t]{2}{*}{ Group } & \multicolumn{3}{|c|}{ Genotype(n) } & \multicolumn{2}{|c|}{ Allele(n) } & \multirow[t]{2}{*}{$P$} & \multicolumn{2}{|c|}{ Dominant } & \multirow[t]{2}{*}{$P$} & \multicolumn{2}{|c|}{ Recessive } & \multirow[t]{2}{*}{$P$} \\
\hline & & MM & $\mathrm{Mm}$ & $\mathrm{mm}$ & $M$ & $\mathrm{~m}$ & & MM & $\begin{array}{l}\mathrm{Mm} \\
+ \\
\mathrm{mm}\end{array}$ & & $\begin{array}{l}\mathrm{MM} \\
+ \\
\mathrm{Mm}\end{array}$ & $\mathrm{mm}$ & \\
\hline \multirow[t]{3}{*}{ rs10494366 } & control & 188 & 101 & 59 & 477 & 219 & & 188 & 160 & & 289 & 59 & \\
\hline & $\begin{array}{l}\text { ICD } \\
\text { patients }\end{array}$ & 43 & 23 & 20 & 109 & 63 & 0.195 & 43 & 43 & 0.503 & 66 & 20 & 0.175 \\
\hline & $\begin{array}{l}\text { appropriate } \\
\text { therapies }\end{array}$ & 12 & 11 & 9 & 35 & 29 & 0.024 & 12 & 20 & 0.073 & 23 & 9 & 0.115 \\
\hline \multirow[t]{3}{*}{ rs12143842 } & control & 179 & 123 & 46 & 481 & 215 & & 179 & 169 & & 302 & 46 & \\
\hline & $\begin{array}{l}\text { ICD } \\
\text { patients }\end{array}$ & 42 & 26 & 18 & 110 & 62 & 0.194 & 42 & 44 & 0.666 & 68 & 18 & 0.071 \\
\hline & $\begin{array}{l}\text { appropriate } \\
\text { therapies }\end{array}$ & 14 & 12 & 6 & 40 & 24 & 0.276 & 14 & 18 & 0.405 & 26 & 6 & 0.384 \\
\hline \multirow[t]{3}{*}{ rs12567209 } & control & 257 & 72 & 19 & 586 & 110 & & 257 & 91 & & 329 & 19 & \\
\hline & $\begin{array}{l}\text { ICD } \\
\text { patients }\end{array}$ & 60 & 19 & 7 & 139 & 33 & 0.284 & 60 & 26 & 0.445 & 79 & 7 & 0.348 \\
\hline & $\begin{array}{l}\text { appropriate } \\
\text { therapies }\end{array}$ & 23 & 6 & 3 & 52 & 12 & 0.539 & 23 & 9 & 0.808 & 29 & 3 & 0.364 \\
\hline \multirow[t]{3}{*}{ rs16847548 } & control & 176 & 96 & 76 & 448 & 248 & & 176 & 172 & & 272 & 76 & \\
\hline & $\begin{array}{l}\text { ICD } \\
\text { patients }\end{array}$ & 40 & 27 & 19 & 107 & 65 & 0.598 & 40 & 46 & 0.500 & 67 & 19 & 0.959 \\
\hline & $\begin{array}{l}\text { appropriate } \\
\text { therapies }\end{array}$ & 16 & 9 & 7 & 41 & 23 & 0.961 & 16 & 16 & 0.950 & 25 & 7 & 0.996 \\
\hline
\end{tabular}

\section{Comparisons Of Clinical Characteristics In Three Genotypes of Rs10494366}

Subsequently, all patients were divided into three groups according to genotypes of the significant SNP rs10494366. The number was 17, 35, 34 for TT, GT, GG genotype, respectively. There was no significant difference in CHD and DCM in three genotypes. The distribution of other cardiovascular diseases such as HCM, ARVC, and LQT syndrome also did not show significant difference in each group. However, the distribution of DM displayed differently in three groups (11, 5, 13 for TT, GT, GG genotype, respectively). Other clinical characteristic data revealed no significant difference as listed in Table 3. 
Table 3

Baseline characteristics of patients in the present study according to genotypes of rs10494366.

\begin{tabular}{|c|c|c|c|c|}
\hline Clinical Characteristic & $\mathrm{TT}(\mathrm{n}=17)$ & GT(n= 35) & $G G(n=34)$ & $P$ values \\
\hline Age, y, mean \pm SD & $55.35 \pm 14.06$ & $53.89 \pm 11.98$ & $52.32 \pm 11.87$ & 0.698 \\
\hline Female, n (\%) & $10(58.82)$ & $15(42.86)$ & $16(47.06)$ & 0.555 \\
\hline Body mass index, $\mathrm{kg} / \mathrm{m}^{2}$, mean $\pm \mathrm{SD}$ & $28.55 \pm 5.65$ & $28.06 \pm 5.57$ & $28.83 \pm 4.33$ & 0.819 \\
\hline Coronary heart disease, n(\%) & $10(58.82)$ & $17(48.57)$ & $12(35.29)$ & 0.249 \\
\hline Dilated cardiomyopathy, n(\%) & $4(23.53)$ & $14(40.00)$ & $16(47.06)$ & 0.268 \\
\hline Other cardiovascular disease, $\mathrm{n}(\%)$ & $3(17.65)$ & $4(11.43)$ & $6(17.65)$ & 0.731 \\
\hline Hypertension, n(\%) & $8(47.06)$ & $13(37.14)$ & $16(47.06)$ & 0.659 \\
\hline Diabetes mellitus, n(\%) & $11(64.71)$ & $5(14.29)$ & $13(38.24)$ & 0.001 \\
\hline \multicolumn{5}{|l|}{ Treatment, n(\%) } \\
\hline ACEI/ARB & $7(41.18)$ & 17(48.57) & $20(58.82)$ & 0.456 \\
\hline Beta-blocker & $13(76.47)$ & $27(77.14)$ & $27(79.41)$ & 0.962 \\
\hline Amiodarone & $7(41.18)$ & $14(40.00)$ & 14(41.18) & 0.994 \\
\hline \multicolumn{5}{|l|}{ Electrocardiology parameters } \\
\hline CLBBB, n(\%) & $10(58.82)$ & 17(48.57) & $16(47.06)$ & 0.713 \\
\hline QRS duration, $\mathrm{ms}$, mean $\pm \mathrm{SD}$ & $141.71 \pm 26.34$ & $130.66 \pm 22.38$ & $133.44 \pm 26.01$ & 0.318 \\
\hline $\operatorname{LVEF}(\%)$, mean \pm SD & $37.29 \pm 9.27$ & $38.20 \pm 10.80$ & $40.65 \pm 11.08$ & 0.487 \\
\hline
\end{tabular}

\section{Comparisons of the ratio of patients suffered from VA episodes and ICD therapies in different genotypes}

At first, we compared the ratio of patients suffered from VA episodes and ICD therapies in different genotype under different genotype model (Table 4). The ratio showed significant different in shocks at 3,12 months after implantation with $P$ of 0.020 and 0.042 , respectively. The significant results also emerged under recessive model of TT genotype $(P=0.023, P=0.024$, respectively). At the end of the follow-up, TT genotype showed significant association with ICD shock under recessive model $(P=$ 0.038) and correlation trend under other two models. 
Table 4

Chi-square test of the ratio of patients suffered from rapid ventricular arrhythmias and ICD therapies in different genotypes under different genotype models.

\begin{tabular}{|c|c|c|c|c|c|c|c|c|c|c|c|c|}
\hline & \multicolumn{4}{|c|}{3 months } & \multicolumn{4}{|c|}{12 months } & \multicolumn{4}{|c|}{ End up } \\
\hline & RVA & ATP & Shock & APT & RVA & ATP & Shock & APT & RVA & ATP & Shock & APT \\
\hline $\begin{array}{l}\text { TT } \\
\text { (17) }\end{array}$ & 7 & 6 & 4 & 6 & 12 & 9 & 7 & 9 & 14 & 9 & 9 & 10 \\
\hline $\begin{array}{l}\text { GT } \\
(35)\end{array}$ & 7 & 5 & 2 & 5 & 9 & 7 & 6 & 7 & 16 & 11 & 9 & 11 \\
\hline $\begin{array}{l}\text { GG } \\
\text { (34) }\end{array}$ & 6 & 5 & 0 & 5 & 11 & 8 & 2 & 8 & 17 & 11 & 4 & 11 \\
\hline$P 1$ & 0.335 & 0.297 & 0.020 & 0.297 & 0.126 & 0.188 & 0.042 & 0.188 & 0.417 & 0.567 & 0.063 & 0.418 \\
\hline$P 2$ & 0.428 & 0.531 & 0.081 & 0.531 & 0.608 & 0.580 & 0.051 & 0.580 & 0.703 & 0.691 & 0.061 & 0.608 \\
\hline$P 3$ & 0.143 & 0.119 & 0.023 & 0.119 & 0.047 & 0.071 & 0.024 & 0.071 & 0.191 & 0.287 & 0.038 & 0.187 \\
\hline
\end{tabular}

Distribution of VA episodes and ICD therapies in three genotypes and the correlation of them with genotypes

Table 5 displayed the distribution of VA episodes and ICD therapies in three genotypes and the correlation of them with different genotypes. Frequencies of VA episodes and ATP after 3 months implantation in three genotypes showed no significant difference $(P=0.103)$, while the distributions of shocks and appropriate therapies in three groups were different $(P=0.011 ; P=0.030$, respectively). The correlation of TT genotype and ICD shock was significant $(P=0.004)$ and the correlation coefficient was 0.308 . Similarly, the distributions of shocks and appropriate therapies in three genotypes at 1 year showed significant $(P=0.006 ; P=$ 0.012 , respectively) and TT genotype correlated with ICD shock $(P=0.003)$. The distributions of shocks at the end of follow-up time also showed significant with $P=0.007$, while the distributions of appropriate therapies were not $\operatorname{significant}(P=0.064)$. ICD shocks at the end of follow-up were correlate with TT genotype with the correlation coefficient of $0.321(P=0.003)$. But the appropriate therapies did not show correlation with rs10494366 correspondingly. 
Table 5

Distribution of rapid ventricular arrhythmias and ICD therapies in three genotypes and the correlation of them with genotypes at three follow-up times.

\begin{tabular}{|llllllllllllll|}
\hline \multicolumn{3}{|c}{ 3 months } & \multicolumn{9}{c}{12 months } & \multicolumn{7}{c|}{ End up } \\
\hline & RVA & ATP & Shock & APT & RVA & ATP & Shock & APT & RVA & ATP & Shock & APT \\
\hline TT & 61 & 60 & 14 & 74 & 105 & 117 & 25 & 142 & 152 & 134 & 40 & 174 \\
\hline GT & 54 & 23 & 4 & 27 & 115 & 98 & 15 & 83 & 199 & 119 & 29 & 148 \\
\hline GG & 19 & 14 & 0 & 14 & 82 & 59 & 7 & 66 & 144 & 102 & 16 & 118 \\
\hline F & 2.337 & 3.099 & 4.737 & 3.660 & 1.954 & 2.647 & 5.533 & 4.637 & 2.069 & 1.989 & 5.250 & 2.837 \\
\hline P1 & 0.103 & 0.050 & 0.011 & 0.030 & 0.148 & 0.077 & 0.006 & 0.012 & 0.133 & 0.143 & 0.007 & 0.064 \\
\hline P2 & 0.061 & 0.110 & 0.004 & 0.100 & 0.047 & 0.053 & 0.003 & 0.051 & 0.053 & 0.167 & 0.003 & 0.074 \\
\hline
\end{tabular}

RVA: rapid ventricular arrhythmia; ATP: anti-tachycardia pacing; APT: appropriate therapies; $F$ : the statistics of ANOVA; $P 1$ for the AVONA of the comparison among the frequencies in three genotypes; $P 2$ for the bivariate correlation of genotypes and the frequencies of episodes.

\section{Comparison Of Cumulative Probability of Survival Classified By Genotypes}

Furthermore, patients with TT genotype underwent worse survival outcome than other two genotypes revealed by Kaplan-meier curve of cumulative probability of survival classified by genotypes of rs10494366 (Fig. 1). The $P$ value was 0.022 assessed by Log Rank method. At the end of follow-up time, the overall mortality of the present study population was $24.41 \%$, while in patients with TT genotype was $47.06 \%$ as revealed by the Kaplan-meier curve. Moreover, after adjusting for CHD, DCM, diabetes mellitus, QRS duration and LVEF, HR of TT genotype for SCD was higher (HR $=2.956, P=0.037)$ compared with GG genotype.

\section{Discussion}

The present study was designed to assess the effect of common variants in NOS1AP on patients receiving an ICD for secondary prevention. We firstly performed a case-control study to investigate the relation between hot-spot SNPs (rs12143842, rs10494366, rs12567209 and rs16847548) in NOS1AP and ICD patients. We found SNP rs10494366 show significant association with ICD patients who suffered appropriate therapies assuming allele model. Then we performed a prospective study to evaluate the clinical value of rs10494366 in these patients. The results of this study indicated that TT genotype of rs 10494366 may play an important role in RVA episodes and shocks recorded by ICD as well as SCD in ICD-treated patients for the first time.

Patients with ICD for secondary prevention yield great risk in recurrence of live-threatening malignant arrhythmia [9]. In the present research, 23.26\% patients suffered from RVA after 3 months of implantation, and among them, $80 \%$ experienced ATP or shock treatment (Fig. 2). At the end of follow-up, the overall RVA episodes were 495 and $54.65 \%$ patients underwent RVA, among which, $68.09 \%$ delivered appropriate therapies. There were $26.74 \%$ patients experienced 85 shocks totally. At the three scheduled follow-up times, we detected not only significant difference among three genotypes on the distribution of ICD shocks and appropriate therapies, but apparently also the correlation of rs10494366 and shocks. Rapid sustained VT failed to termination after ATPs and VF, which represent more unstable status of electrophysiology of ventricular myocytes [10], will both trigger shocks delivered by ICD. Obviously, cardiac death frequently occurred accompanied with ICD therapies. Prior studies showed that most sudden deaths among ICD patients resulted from electromechanical dissociation occurring after appropriately detected and treated VAs [11]. Small size sample clinical data also displayed VA episodes and unsuccessful defibrillations prior to death as interrogated at the time of autopsy $[12,13]$. In the present study, patients who carried TT genotype suffered more appropriate therapies including ICD shocks than other genotypes, thus with poor prognosis. 
The total cardiovascular mortality at the end of follow-up time was identified as $24.41 \%$, which was a little higher than previous studies performed in Veterans Administration trial [14] after 3 years of implantation. These different outcomes probably partly attributed to low proportion of optimal medical therapy for VT, heart failure and coronary artery disease. Furthermore, the present study revealed the probability of survival $(52.94 \%)$ was lower in TT genotype. TT genotype showed greater risk for SCD under both kaplan-meier analysis and cox regression analysis. From all the above, TT genotypes of rs10494366 may play a part role in the occurrence and maintenance of RVAs and showed correlation to shocks in ICD recipients with different etiologies.

Rs10494366, which located in the intron region of NOS1AP, is a regulator of neuronal NOS $[1,15]$. Many researches verified rs10494366 in NOS1AP gene was associated with cardiac repolarization including prolonged QT interval and sudden death in independent populations [16-24]. SNPs, which located in noncoding region, could influence transcriptional by exerting cis-acting elements. Vitro experiments has elucidated potential transcriptional effects of certain common variants in NOS1AP on schizophrenia [25]. Besides, Chang et al [26] found that overexpression of the NOS1AP gene product in isolated guinea pig myocytes causes attenuation of L-type calcium current, a slight increase in rapid delayed rectifier current $I_{\mathrm{K} r}$, and shortening of action potentials. Though the potential transcriptional effects of NOS1AP variants on gene expression in heart are not clearly known, these observations might explain our findings to a certain extent.

\section{Study Limitations}

The present study is subject to several limitations. First, in some patients who have poor compliance of optimal treatment, electric storm might readily deliver without adjusting the ICD parameters in time, which could increase the weight of statistical validity slightly. But in other words, the susceptibility to electric storm in these patients revealed the potential arrhythmogenic of this allelic variant. Second, there is hitherto no standard definition of VT/VF detection and therapy zone for ICD. Though we set a broad criterion, VT below the lower limit frequency, which is unable to detected, may inevitably dilute the impact of rs 10494366 on VT episodes or arrhythmic death. Besides, to provide optimal therapies delivered by ICD, we adjusted the parameters of ICD in four patients according to documented arrhythmias resulted from deterioration of physical condition, which probably influence the ATP or shock but would achieve more appropriate therapies on the other hand. However, considering the relatively small number of patients in these set, the influence could be negligible. Moreover, the retrospective analysis in Japanese found left ventricular diastolic diameter $\geq 60 \mathrm{~mm}$ and the presence of non-sustained ventricular tachycardia before implantation were independent predictors of appropriate ICD therapy [27]. As the lack of these data, we are unable to bring into these indicators for analysis.

\section{Conclusion}

To our knowledge, the present study evaluated SNPs in NOS1AP in patients with ICD for secondary prevention for the first time. The association analysis revealed that minor allele of rs 10494366 was associated with appropriate therapies in ICD patients. The correlation between rs10494366 and clinical events detected by ICD revealed that TT genotype of NOS1AP may play a role on ICD shock and SCD. Thus, TT genotype of rs10494366 may be clinically useful for risk stratification of RVA in patients receiving ICD for secondary prevention. However, researches focus on the functional test and large sample population are absolutely needed.

\section{Abbreviations}

NOS1AP: Nitric oxide synthase 1 adaptor protein; ICD:Implantable cardioverter defibrillator; SCD: Sudden cardiac death; VA:Ventricular arrhythmia; RVA:Rapid ventricular arrhythmia; VT:Ventricular tachycardia; VF:Ventricular fibrillation; CHD:Coronary heart disease; DCM:Dilated cardiomyopathy; HCM:Hypertrophic cardiomyopathy; ARVC:Arrhythmogenic right ventricular cardiomyopathy; PCR:Polymerase chain reaction; CL:Circumference length; ATP:Anti-tachycardia pacing

\section{Declarations}

\section{Acknowledgments}

Page 10/14 
We thank all the researchers who participated in this work.

\section{Funding}

This work was supported by Henan Province Medical Science and Technology Tackling Project (NO.201303134); Henan Medical Science and Technology Research Project (No:201702163); Medical Science and Technique Program of He'nan Provincial Health and Family Planning Commission (NO.201602214); Science and Technique Program of He'nan Provincial Department of Science and Technology (NO.172102310065).

\section{Availability of data and materials}

The datasets used and/or analysed during the current study are available from the corresponding author on reasonable request.

\section{Authors' contributions}

Conception and design of the study: XZ, SZ, YZ and CG. Data collection: XZ, WS, KC and JM. Data analysis: XZ, SZ, XT and YZ. Manuscript writing: $X Z, S Z$ and $Y X$. Critical revision: $Y Z$ and $C G$. All authors have read and approved the final manuscript.

\section{Ethics approval and consent to participate}

The study was approved by the Ethics Committee of Henan provincial people's hospital. All the patients enrolled in the study signed written informed consent.

\section{Consent for publication}

Not applicable.

\section{Competing interests}

The authors declare no conflicts of interest.

\section{References}

1. Arking DE, Pfeufer A, Post W, et al. A common genetic variant in the NOS1 regulator NOS1AP modulates cardiac repolarization. Nat Genet. 2006;38:644-51.

2. Kao WH, Arking DE, Post W, et al. Genetic variations in nitric oxide synthase 1 adaptor protein are associated with sudden cardiac death in US white community-based populations. Circulation. 2009;119:940-51.

3. Earle N, Yeo HD, Pilbrow A, et al. Single nucleotide polymorphisms in arrhythmia genes modify the risk of cardiac events and sudden death in long QT syndrome. Heart Rhythm. 2014;11:76-82.

4. Kolder IC, Tanck MW, Bezzina CR. Common genetic variation modulating cardiac ECG parameters and susceptibility to sudden cardiac death. J Mol Cell Cardiol. 2012;52(3):620-9.

5. ACC/AHA/HRS

Epstein AE, DiMarco JP, Ellenbogen KA, et al. ACC/AHA/HRS 2008 Guidelines for Device-Based Therapy of Cardiac Rhythm Abnormalities: a report of the American College of Cardiology/American Heart Association Task Force on Practice Guidelines (Writing Committee to Revise the ACC/AHA/NASPE 2002 Guideline Update for Implantation of Cardiac Pacemakers and Antiarrhythmia Devices): developed in collaboration with the American Association for Thoracic Surgery and Society of Thoracic Surgeons. Circulation 2008;117:e350-408.

6. Cleland JG, Daubert JC, Erdmann E, et al. The effect of cardiac resynchronization on morbidity and mortality in heart failure. N Engl J Med. 2005;352:1539-49.

7. Elliott P, Andersson B, Arbustini E, et al. Classification of the cardiomyopathies: a position statement from the European Society Of Cardiology Working Group on Myocardial and Pericardial Diseases. Eur Heart J. 2008;29:270-6. 
8. Priori SG, Aliot E, Blomstrom-Lundqvist C, et al. Task Force on Sudden Cardiac Death of the European Society of Cardiology. Eur Heart J. 2001;22:1374-450.

9. Miller JD, Yousuf O, Berger RD. The implantable cardioverter-defibrillator: An update. Trends Cardiovasc Med. 2015;25:60611.

10. Jalife J. Ventricular fibrillation: mechanisms of initiation and maintenance. Annu Rev Physiol. 2000;62:25-50.

11. Mitchell LB, Pineda EA, Titus JL, Bartosch PM, Benditt DG. Sudden death in patients with implantable cardioverter defibrillators: the importance of post-shock electromechanical dissociation. J Am Coll Cardiol. 2002;39:1323-8.

12. The Antiarrhythmics Versus Implantable Defibrillators (AVID). Investigators. A comparison of antiarrhythmic-drug therapy with implantable defibrillators in patients resuscitated from near-fatal ventricular arrhythmias. N Engl J Med. 1997;337:1576-83.

13. Sinha SK, Crain B, Flickinger K, et al. Clinical Inferences of Cardiovascular Implantable Electronic Device Analysis at Autopsy. J Am Coll Cardiol. 2016;68:1255-64.

14. Chan PS, Hayward RA. Mortality reduction by implantable cardioverter-defibrillators in high-risk patients with heart failure, ischemic heart disease, and new-onset ventricular arrhythmia: an effectiveness study. J Am Coll Cardiol. 2005;45:1474-81.

15. Tomas M, Napolitano C, De Giuli L, et al. Polymorphisms in the NOS1AP gene modulate QT interval duration and risk of arrhythmias in the long QT syndrome. J Am Coll Cardiol. 2010;55:2745-52.

16. Tobin MD, Kahonen M, Braund P, et al. Gender and effects of a common genetic variant in the NOS1 regulator NOS1AP on cardiac repolarization in 3761 individuals from two independent populations. Int J Epidemiol. 2008;37:1132-41.

17. Lehtinen AB, Newton-Cheh C, Ziegler JT, et al. Association of NOS1AP genetic variants with QT interval duration in families from the Diabetes Heart Study. Diabetes. 2008;57:1108-14.

18. Raitakari OT, Blom-Nyholm J, Koskinen TA, et al. Common variation in NOS1AP and KCNH2 genes and QT interval duration in young adults. The Cardiovascular Risk in Young Finns Study. Ann Med. 2009;41:144-51.

19. Pfeufer A, Sanna S, Arking DE, et al. Common variants at ten loci modulate the QT interval duration in the QTSCD Study. Nat Genet. 2009;41:407-14.

20. Arking DE, Khera A, Xing C, et al. Multiple independent genetic factors at NOS1AP modulate the QT interval in a multi-ethnic population. PLoS One. 2009;4:e4333.

21. Eijgelsheim M, Aarnoudse AL, Rivadeneira F, et al. Identification of a common variant at the NOS1AP locus strongly associated to QT-interval duration. Hum Mol Genet. 2009;18:347-57.

22. Lu J, Hu C, Hu W, et al. A common variant of NOS1AP is associated with QT interval duration in a Chinese population with Type 2 diabetes. Diabet Med. 2010;27:1074-9.

23. Smith JG, Avery CL, Evans DS, et al. Impact of ancestry and common genetic variants on QT interval in African Americans. Circ Cardiovasc Genet. 2012;5:647-55.

24. Zang X, Li S, Zhao Y, et al. Systematic Meta-Analysis of the Association Between a Common NOS1AP Genetic Polymorphism, the QTc Interval, and Sudden Death. Int Heart J. 2019 Sep;27(5):1083-90. 60(.

25. Wratten NS, Memoli H, Huang Y, et al. Identification of a schizophrenia-associated functional noncoding variant in NOS1AP. Am J Psychiatry. 2009;166:434-41.

26. Chang KC, Barth AS, Sasano T, et al. CAPON modulates cardiac repolarization via neuronal nitric oxide synthase signaling in the heart. Proc Natl Acad Sci USA. 2008;105:4477-82.

27. An Y, Ando K, Soga Y, et al. Mortality and predictors of appropriate implantable cardioverter defibrillator therapy in Japanese patients with Multicenter Automatic Defibrillator Implantation Trial II criteria. J Arrhythm. 2017;33:17-22.

\section{Figures}




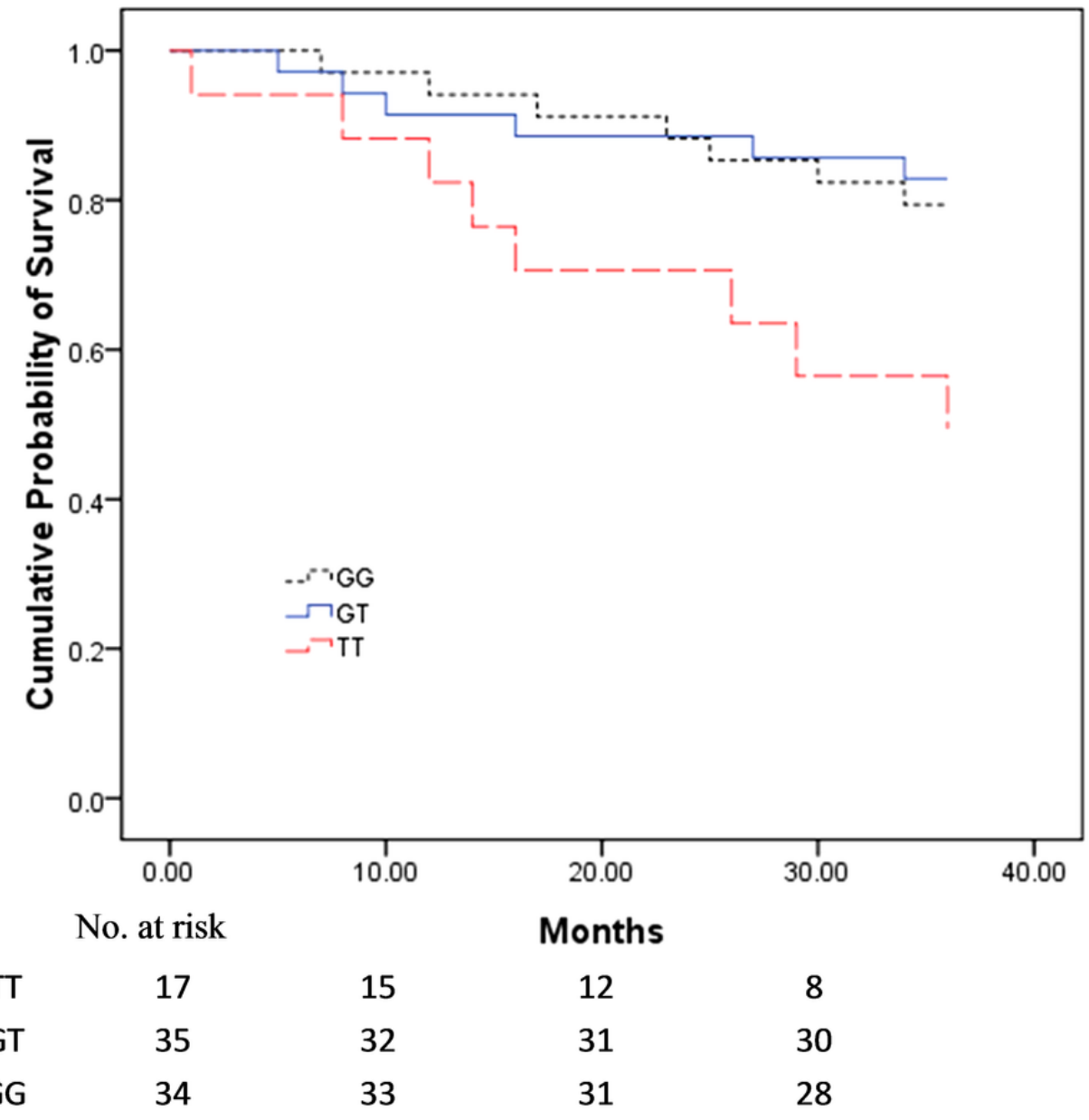

Figure 1

Kaplan-meier curve of the cumulative probability of survival classified by genotypes of rs10494366. 


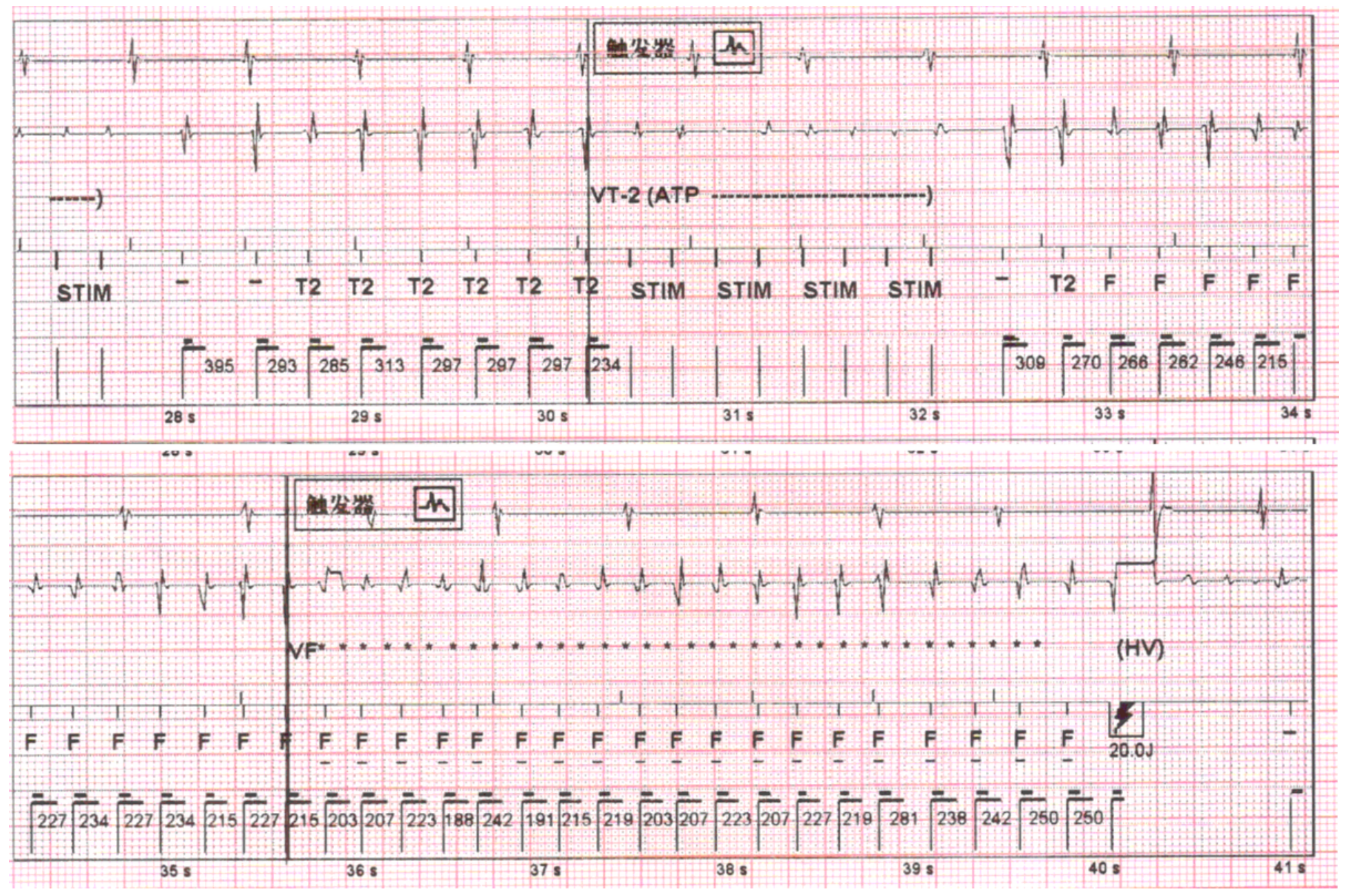

Figure 2

One case of ventricular tachycardia CL coincided fast VT zone (T2), ICD shock delivered after three tracings of ATP(STIM) failure.

\section{Supplementary Files}

This is a list of supplementary files associated with this preprint. Click to download.

- Additionalfile1.docx 